\title{
Editorial: Bildung in Corona-Zeiten
}

\author{
Marcus Hasselhorn • Ingrid Gogolin
}

Angenommen: 9. April 2021 / Online publiziert: 26. April 2021

(C) Der/die Autor(en) 2021

Seit Mitte März 2020 hält die Corona-Pandemie Europa und - mit zeitlichen Variationen - die ganze Welt in Atem. Es gibt kaum mehr einen Lebensbereich, der davon unberührt geblieben wäre. Die Auswirkungen auf das Bildungswesen sind massiv: Schulen und Kitas wurden ganz oder teilweise geschlossen, Universitäten haben auf digitale Lehre ohne Präsenzveranstaltungen umgestellt. Bildungsteilnehmende - und im Falle von Kindern auch ihre Eltern - sowie das pädagogisch verantwortliche Personal mussten von heute auf morgen notgedrungen ihre vertrauten Routinen aufgeben. Digitale Lehr-Lern-Formate erfuhren eine nie geahnte Konjunktur. Die Folgen sind derzeit bestenfalls begrenzt zu erahnen.

Wie ändern sich Einstellungen zu Bildung? Wie erleben Bildungsteilnehmende und deren Erziehungsberechtigte die neuen häuslichen Lehr-Lernformate? Welche Kosten entstehen für Bildungsteilnehmende und die Gesellschaft? Welche Risiken und Chancen entstehen für individuellen Bildungserfolg und die Qualität von Bildungsangeboten?

Dies sind nur einige der Fragen zu den Folgen der Pandemie im Bildungswesen. In den Bildungs- und Erziehungswissenschaften wurden sehr schnell einige Studien zu Fragen dieser Art initiiert. Andere Quellen zur Bearbeitung von Detailfragen ergeben sich aus bereits vorher angelaufenen empirischen Projekten, denen die Pandemie in

Prof. Dr. M. Hasselhorn ( $\square)$

DIPF । Leibniz-Institut für Bildungsforschung und Bildungsinformation, Rostocker

Str. 6, 60323 Frankfurt am Main, Deutschland

E-Mail: hasselhorn@dipf.de

Prof. Dr. Dr. h.c. mult. I. Gogolin

Fakultät für Erziehungswissenschaft, Fachbereich 1, Allgemeine, Interkulturelle und International vergleichende Erziehungswissenschaft, Universität Hamburg, Von-Melle-Park 8, 20146 Hamburg, Deutschland

E-Mail: gogolin@uni-hamburg.de 
die Quere kam, die aber teilweise auch Möglichkeiten für Corona-Folgen-Analysen eröffnen.

Die Zeitschrift für Erziehungswissenschaft hat daher im Sommer letzten Jahres einen Aufruf gestartet, Beiträge zu diesem Themenfeld einzureichen. Die Resonanz war erfreulich groß. Die ersten neun Beiträge, die zu dieser Thematik das Begutachtungsverfahren erfolgreich durchlaufen haben, sind in diesem Themenschwerpunktheft zu finden.

Das Schwerpunktheft startet mit einem systematischen Überblick über die von Christoph Helm, Stephan Huber und Tina Loisinger in den deutschsprachigen Ländern identifizierten 97 Online-Befragungen, die in der Zeit vom 24. März bis 11. November 2020 zum Thema Schulsituation und Lehren und Lernen während der Corona-Pandemie durchgeführt wurden. Die Autorengruppe listet jedoch nicht nur die durchgeführten Befragungen, sondern versucht auch, die zentralen Erkenntnisse daraus zu beschreiben und die wissenschaftliche Qualität der erhobenen Daten kritisch einzuordnen.

Der Beitrag von Franziska Cohen, Elisa Oppermann und Yvonne Anders basiert auf einer bundesweiten Onlinebefragung von pädagogischen Fachkräften aus Kindertageseinrichtungen, bei der die (digitale) Elternzusammenarbeit in von der Pandemie beeinträchtigten Zeiten thematisiert wurde. Ein Ergebnis ist, dass Fachkräfte, die sich selbst als verantwortlich für die Elternzusammenarbeit sahen, auch eher in dieser Zeit Kontakt mit den Eltern aktiv aufnahmen. Wie dies geschah, hing entscheidend von den persönlichen Einstellungen zu digitalen Medien, den erwarteten Reaktionen der Eltern auf digitale Kontaktangebote und der verfügbaren technischen Unterstützung ab.

Die Sonderbefragung teilnehmender Eltern schulpflichtiger Kinder des SozioOekonomischen Panels (SOEP) zum Thema Corona bildet die Datenbasis des dritten Beitrags von Sabine Zinn und Michael Bayer zur subjektiven Belastung der Eltern durch die Beschulung ihrer Kinder zu Hause. Im Durchschnitt wurden eher mäßige Belastungen wahrgenommen. Besonders hoch war die Wahrnehmung der Belastung bei Eltern mit niedrigem Bildungsabschluss und bei alleinerziehenden Eltern, wenn diese erwerbstätig waren.

Daten eines sehr ungewöhnlichen Datensatzes liegen dem Beitrag von Friederike Blume, Andrea Schmidt, Andrea C. Kramer, Florian Schmiedek und Andreas B. Neubauer zugrunde. In einer Online-Befragung schätzten die Eltern von Schulkindern die Fähigkeit ihrer Kinder zur Selbstregulation ein. Anschließend machten die Eltern 21 Tage lang Angaben über die täglichen Aufgabenschwierigkeiten, die Lernfreude und das selbstständige Lernen ihrer Kinder. Es zeigte sich eine positive Korrelation zwischen der eingeschätzten Fähigkeit der Kinder zur Selbstregulation und der Selbstständigkeit des täglichen Lernens im häuslichen Kontext.

Selbstreguliertes Lernen ist auch Gegenstand des fünften Beitrags von Elisabeth Rosa Pelikan, Marko Lüftenegger, Julia Holzer, Selma Korlat, Christiane Spiel und Barbara Schober. Befragt wurden mehr als 2600 österreichische Sekundarstufenschülerinnen und -schüler zum selbstregulierten Lernen, intrinsischer Motivation, passiver Prokrastination und selbstwahrgenommener Kompetenz. Strukturgleichungsanalysen zeigen, dass Schülerinnen und Schüler mit hoher selbstwahrgenommener Kompetenz mehr selbstreguliertes Lernen berichten und sich in stärke- 
rem Maße intrinsische Motivation zuschreiben. Qualitative Zusatzanalysen ergaben, dass diejenigen mit hoher selbstwahrgenommener Kompetenz besonders gut mit den Herausforderungen des Distanzunterricht zurechtkamen und weniger Unterstützung durch Erwachsene benötigten.

Der Beitrag von Andreas Sander, Laura Christina Schäfer und Stefanie van Ophuysen basiert auf einer standardisierten Onlinebefragung der Eltern von Sekundarstufenschülerinnen und -schülern zur elterlichen Unterstützung während des Distanzunterrichts. Regressionsanalytische Befunde sprechen für eine weitgehend adaptive Lernbegleitung der Eltern, die besonders intensiv ausfällt bei jüngeren männlichen Schülern mit ungünstigem Lernverhalten. Auch die Lernbegleitung durch die Lehrkräfte ist prädiktiv für das elterliche Verhalten, indem engagierte Begleitung durch die Lehrkraft gerade bei Familien mit nicht-gymnasialer Schulbildung der Eltern zu verstärkter Unterstützung des Kindes beiträgt.

Tim Fütterer, Emely Hoch, Kathleen Stürmer, Andreas Lachner, Christian Fischer und Katharina Scheiter analysierten für den siebten Beitrag dieses Schwerpunktthemas die digitale Kommunikation unter Lehrkräften im „Twitter-Lehrerzimmer“. Mehr als 128.000 Tweets wurden mit computerlinguistischen Methoden ausgewertet. Es zeigte sich, dass Themen zum digitalen Unterricht zwar bereits vor den Schulschließungen thematisiert wurden, während der Schulschließungen jedoch häufiger und breiter. Qualitative Inhaltsanalysen ergaben, dass das Twitter-Lehrerzimmer für gegenseitige Unterstützung sowie den Austausch über drängende Herausforderungen genutzt wurde, wie etwa die Verfügbarkeit geeigneter datenschutzkonformer Software.

Mit der Sicht von Schülerinnen und Schülern weiterführender Schulen zum Gelingen von Fernunterricht beschäftigt sich der Beitrag von Hannah Jäkel und Ivo Züchner. Ausgehend von durch das Angebots-Nutzungs-Modell inspirierten Überlegungen zum Bedingungsgefüge von Fernbeschulung werden Angaben von Schülerinnen und Schülern weiterführender Schulen zur Bewältigung von „Haus“aufgaben mit besonderem Fokus auf die Rolle digitaler Medien analysiert. Auf der Basis der Befunde wird eine Erweiterung des Angebots-Nutzungs-Modells um digitale Elemente von Infrastrukturen, Kommunikation, Lehrformen sowie der Kompetenzen von Lehrenden und Lernenden.

Im abschließenden Beitrag von Sverker Lindblad, Anders Lindqvist, Caroline Runesdotter und Gun-Britt Wärvik wird der Blick über den deutschsprachigen Tellerrand hinaus nach Schweden gelenkt. Dort gehörte das Offenbleiben der Schulen zur Strategie im Umgang mit der Pandemie. Die Autorengruppe geht in ihrem Beitrag der Frage nach, welche Rolle das Bildungssystem in Schweden gespielt hat, um das gesellschaftliche Leben aufrecht zu erhalten. Dazu werden Regierungserklärungen und Medienberichte analysiert und ein optimistisches Bild zu Potenzialen des Bildungswesens in der Bewältigung der pandemischen Herausforderungen in den Überzeugungen der schwedischen Gesellschaft nachgezeichnet.

Insgesamt beziehen sich die Beiträge dieses Themenschwerpunktes auf Ereignisse, Sichtweisen und Erfahrungen, die eine relativ frühe Phase der Pandemie betreffen. Insofern bildet dieses Themenheft auch Grundlagen für weitere Beobachtungen und Analysen der Entwicklungen in Folge der Pandemie, die gegenwärtig - wie gesagt - noch kaum zu erahnen sind. 
Funding Open Access funding enabled and organized by Projekt DEAL.

Open Access Dieser Artikel wird unter der Creative Commons Namensnennung 4.0 International Lizenz veröffentlicht, welche die Nutzung, Vervielfältigung, Bearbeitung, Verbreitung und Wiedergabe in jeglichem Medium und Format erlaubt, sofern Sie den/die ursprünglichen Autor(en) und die Quelle ordnungsgemäß nennen, einen Link zur Creative Commons Lizenz beifügen und angeben, ob Änderungen vorgenommen wurden.

Die in diesem Artikel enthaltenen Bilder und sonstiges Drittmaterial unterliegen ebenfalls der genannten Creative Commons Lizenz, sofern sich aus der Abbildungslegende nichts anderes ergibt. Sofern das betreffende Material nicht unter der genannten Creative Commons Lizenz steht und die betreffende Handlung nicht nach gesetzlichen Vorschriften erlaubt ist, ist für die oben aufgeführten Weiterverwendungen des Materials die Einwilligung des jeweiligen Rechteinhabers einzuholen.

Weitere Details zur Lizenz entnehmen Sie bitte der Lizenzinformation auf http://creativecommons.org/ licenses/by/4.0/deed.de. 